\title{
How to Improve Progress in Scoliosis Research
}

\author{
Keith M. Bagnall \\ United Arab Emirates University \\ United Arab Emirates
}

\section{Introduction}

Since the time of Hippocrates, patients with abnormally-curved spines have been observed and their condition well documented. It is recognized that there are several different types of scoliosis identified by the age of initial appearance and the curves can take several different forms including single and double, directed to the left or to the right, spanning different vertebral levels, and with differing degrees of curvature. Whatever the form, the curves are 3-dimensional and involve vertebral rotation as an additional defining characteristic. The most common form of scoliosis is adolescent idiopathic scoliosis (AIS) and refers to the development of abnormal curvature of the vertebral column that becomes evident at the time of puberty. As its name suggests, it also has no apparent cause. AIS represents $80 \%$ of the cases of scoliosis and will be the primary focus of this chapter.

Patients with scoliosis form a small but significant portion of the general population. Many of the curves are sufficiently small to be simply of a cosmetic nature but, nevertheless, are very noticeable and upsetting for the patient who is at an important stage of their adolescent development. Understandably, the patients request treatment and elimination of the curve. However, for the unfortunate few, the curves go beyond being cosmetic and are of such magnitude that the rotation causes the ribs to impinge on the heart and lungs and severely affects cardio-respiratory function (Keim, 1979).

The mechanism behind the development of such curves is both fascinating and complex and has been the focus of much research especially in the last 100 years or so. Unfortunately, in spite of this intense activity, very little is known about scoliosis with any degree of certainty and, quite simply, the return on any research investment has been limited. So much so that if a patient could be identified with $100 \%$ certainty that they will develop scoliosis in the near future then there is little that can be done other than watch its development (see Figure 1). There are many treatment strategies that might be considered (e.g. bracing and exercise) and applied but none seem able to guarantee prevention of the curve development other than the insertion of long metal rods and fusion of the affected vertebrae. This is drastic, invasive treatment and is not acceptable.

If the curve develops sufficiently ( 50 degrees) then the insertion of metal rods is made. While this effectively curtails further curve development it is a drastic procedure that can surely be replaced by something better and less invasive in this day and age. A better understanding of the aetiology of AIS and the underlying mechanics of curve development 
would lend itself towards the development of improved treatment strategies at earlier stages that would be more effective and far less invasive.

This chapter will focus on the changes that are needed in regards to research into scoliosis so that much greater progress can be made in comparison with the limited progress that has been made so far. Albert Einstein is often quoted as saying that: 'To continue doing the same thing and expecting different results is a sign of madness'. This statement can be used as a platform on which to build.

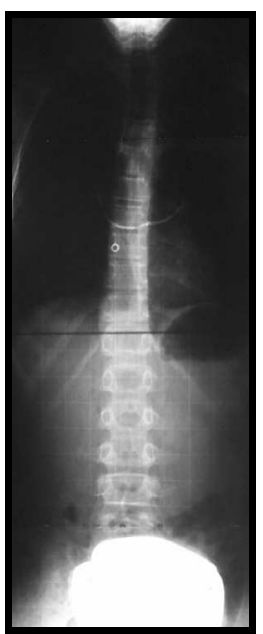

A

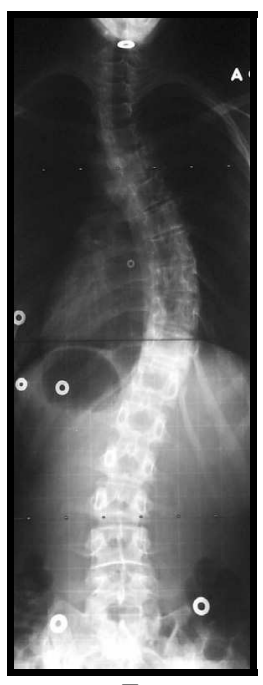

F

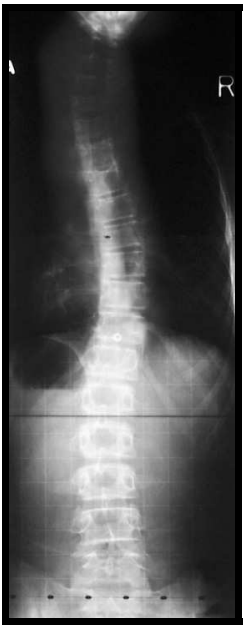

B

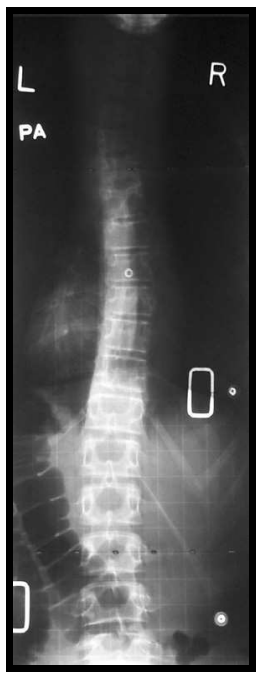

G

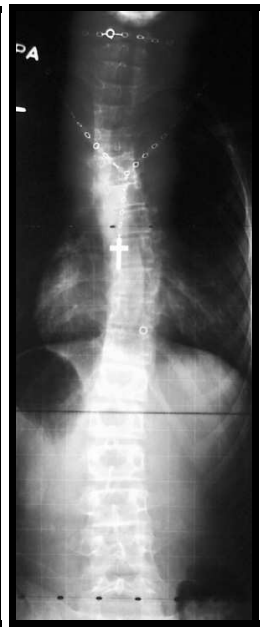

C

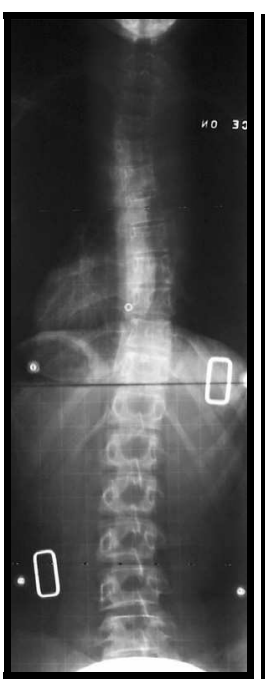

$\mathrm{H}$

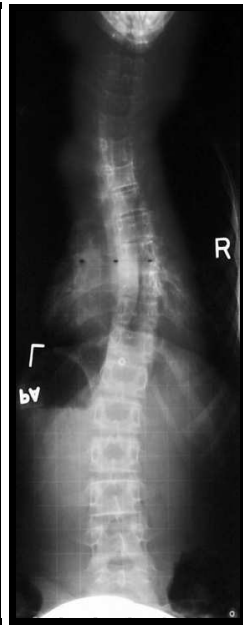

D

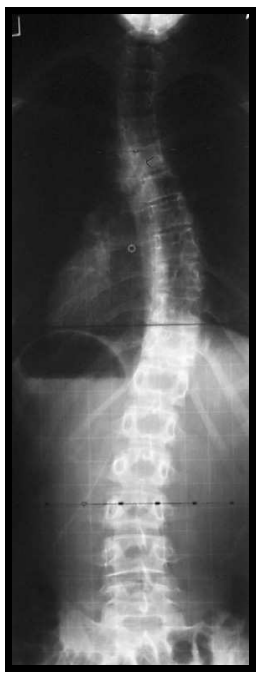

I

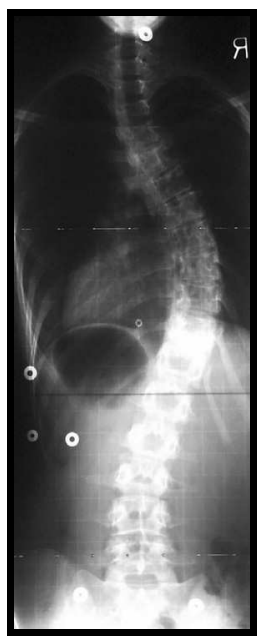

$\mathrm{E}$

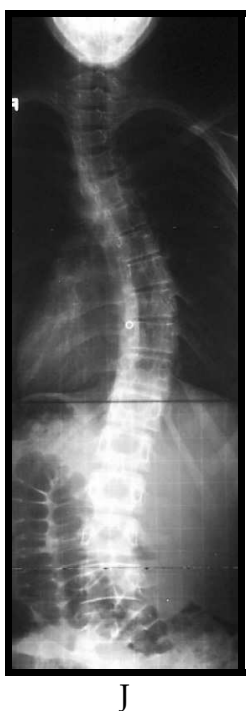



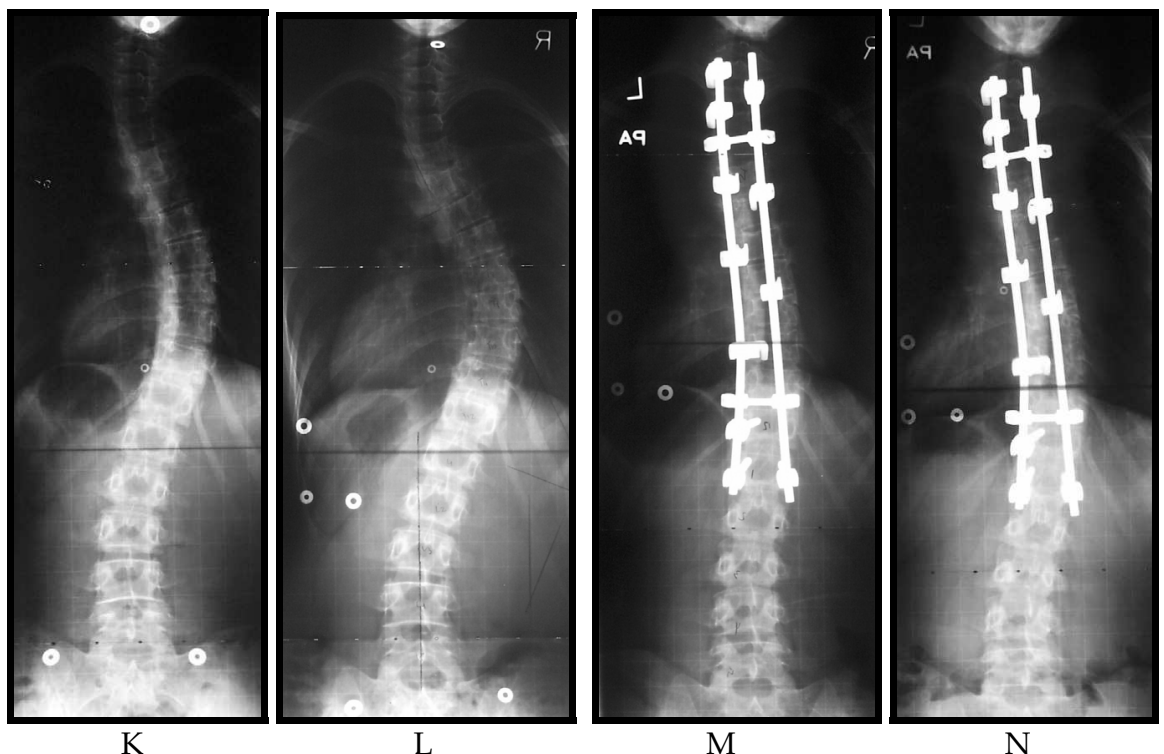

Fig. 1. A series of radiographs showing development of AIS in a young girl.

Such a sequence of radiographs is rare because there is a radiograph available of the times when she did not have an abnormal spinal curve and radiographs which show the early curve development are usually not available. A - radiograph before any curve development. B, C, D, E, and F - curve development over the next two years. $\mathrm{G}$ and $\mathrm{H}$ - reduction of the curve during brace treatment. I, J, K, and L - continued curve development after brace treatment was stopped. $\mathrm{M}$ and $\mathrm{N}$ - radiographs after surgery for the insertion of metal supporting rods. This sequence of radiographs illustrates the lack of effective treatment strategies currently available to deal effectively with patients who have AIS.

\section{What have we learned with certainty from the research conducted into scoliosis in the past 100 years?}

The vertebral column is a complicated structure consisting of many interactive and overlapping components. Using many different techniques, research into AIS appears to have explored many, if not all, of the components of the vertebral column in an effort to discover the underlying mechanism of any abnormal development. Unfortunately the results of such research have often been contradictory and confusing with those indicating a positive result being counteracted by results from similar research showing either equivalent negative or neutral conclusions. For example, different research projects measuring the levels of growth hormone during development of AIS have shown negative influence, neutral influence and positive influence all with seemingly similar patients and experimental protocols.

Research into scoliosis has been intense over the last 100 years but really there are only four basic facts that appear to be beyond dispute: 
- Development of AIS requires the presence of a growth spurt.

- $\quad$ Most $(80 \%)$ of the patients are female.

- Many different kinds of curve can develop and be present within the group of patients

- $\quad$ AIS appears to be familial (present in families)

Although there have perhaps been other less significant findings, it is these four facts that summarize the research findings in relation to the aetiology of AIS during the past 100 years. This is disappointing because observation for just 20 minutes in a scoliosis clinic would provide the same information! The patients would be adolescents undergoing significant growth, most of them would be female, there would be a variety of curves among the patients, and there might well be a relative accompanying the patient who also has the same problem. This lack of specific findings is disappointing but perhaps as a whole they provide us with the motivation, impetus and guidance to change the way that we are approaching the research. They emphasise to us the need to change.

\section{Where to focus efforts}

Figure 2 shows the hypothetical development of a scoliosis curve in a typical patient. When the patient is born there is no obvious curve present and this remains so until approximately the age of 10 years. At this age a curve starts to develop but it is not yet of sufficient magnitude to be noticeable. The curve continues to develop for approximately 18 months, steadily getting more severe. (Because of the nature of the curve development, the length of time for a patient to have a spinal curve before it is noticed is unknown.) It is interesting to note that the patient is unaware of a curve developing with no intrinsic symptoms being manifest. Eventually, 'somebody' notices a curve (usually somebody else - 'stand up straight', 'your shoulders are not level....) and so the patient visits their family physician for advice. An appointment is made with a specialist with an inevitable further delay because of a surfeit of patients. The diagnosis of scoliosis is subsequently confirmed and the curve at this point can get worse, remain the same or improve.

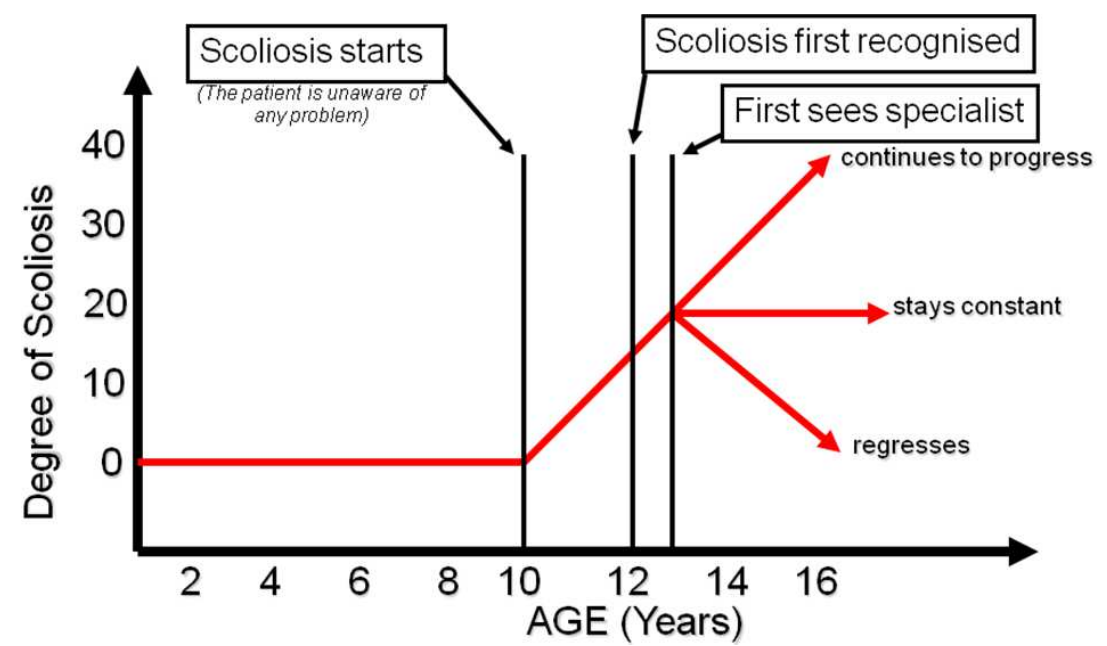

Fig. 2. A graph showing the hypothetical timeline for the development of AIS. 
There is no curve development until approximately the age of 10 years old at which time the curve starts to develop steadily. The curve is not noticed until it is sufficiently severe ( 15 degrees) which has taken possibly 2 years. After seeing a specialist the curve can either continue to progress, stay constant or even regress.

Following this hypothetical pathway, the initiation of treatment of the spinal curve is delayed perhaps 2 years since the inception of curve development. This in itself presents significant difficulties in research:

- When the patient is first seen in the scoliosis clinic, they have had a curve for perhaps 2 years. During this time the underlying cause might well have resolved and the patient might have nothing wrong with them at the time of examination other than having a scoliosis curve.

- Bone is very malleable and responds to extrinsic forces. The presence of a force producing a spinal curve over a period of time leads to vertebral malformation, especially of the vertebral body. This malformation might be such as to not allow the spine to become straight even if the underlying, deforming cause is able to be removed. With a curved spine that has developed over several months, the underlying cause might not be present any more and the presence of the curve and its potential for increase is now a purely biomechanical one with the foundation being malformed vertebrae.

- The period of time that would be very beneficial for research focus is the time when the curve first starts to form. However, this cannot currently be identified because the curve is small and not noticeable at this time. The initial stages in the study of curve development are inevitably made after the curve has been present for a considerable time and not at the critical period when the curve is first initiated.

- This argument is often amplified when differences between patients with scoliosis and normal subjects are examined because patients with advanced scoliosis are often selected for study and their curves are particularly severe. In such cases, the scoliosis has often been present for many years and the biomechanical influence has had a major influence.

By the time the patient is examined in the scoliosis clinic, the underlying cause might no longer be present. Certainly a curve can be demonstrated and it might well be seen to progress over time but the problem might simply be a biomechanical problem. Gathering samples of tissue at this time might well be futile because the patient could be entirely normal. The underlying cause might no longer be present.

The fact that a patient with AIS has had the spinal curve for at least several months (if not years) also presents another major problem for researchers. The development of the curve and its accompanying mechanics has many effects on the tissues of the spine. Separating the effects that have been created by the development of the curve from those that were present before the development of the curve is very difficult. Nevertheless, when the research involves comparison of tissues collected from patients with AIS with similar tissues collected from normal subjects, then this becomes an important issue which is difficult to resolve and clarify. Research into the study of AIS is fraught with separation of 'cause from effect'.

Observation and analysis of the initial stages of scoliosis development are critical to an understanding of the aetiology of AIS and yet they have never been studied because 
patients are identified only after this period. Attempts have been made to identify the early stages of curve development by associating them with ribcage deformity but for this to be visible, the curve has to have been present for a considerable time and any observations are not being made at the critical time when the curve is first initiated.

School-screening studies have shown that the incidence of scoliosis is $\sim 2 \%$ and that development of severe scoliosis requiring surgery is only $3 \%$ of that population. While these studies have been proved to be very valuable, these figures make very difficult the continued observation of a group of normal subjects to observe early curve development and certainly impractical financially. Consequently, a case can be made to identify a population in which the incidence of scoliosis is higher than in the normal population. Perhaps this population can be developed from the relatives of the patients who attend the scoliosis clinic - their siblings and cousins. As AIS is familial, then such a population would have a higher probability of developing AIS. Maybe if this population was then followed closely, more cases in which the early identification of curve development could be found and invaluable data collected.

From Figure 2. it can be seen that there are three areas where efforts should be focused if significant progress is to be made:

- Development of a marker that can predict the future development of scoliosis would be of enormous benefit. Such a marker could be applied at an early age ( $\sim 5$ years of age) so that preparation can be made for appropriate treatment strategies and initial curve development thwarted.

- In combination or separately, a marker that would predict the severity of future curve development would also be of great benefit. When the scoliosis curve is first detected it is not possible to predict future development with accuracy. Currently, the age of the patient, physical maturity, current curve development and rate of development are all taken into account to predict future development but the addition of a definitive marker would be a tremendous advance for diagnosis application.

- There is a need for appropriate strategies to be developed for the treatment of small curves so that they do not develop into major curves. All major curves started off as small curves and so there is a need for the development of treatment strategies that will curtail any further development.

Figure 2 also offers other areas for consideration as major issues for the focus of attention (for example, the development of better treatment strategies for severe curves which do not involve such invasive surgery) but if the three areas outlined above can be resolved then the other areas of interest would be dramatically reduced as a result. If a better understanding of the aetiology of AIS can be obtained, then better treatment strategies can be developed as a consequence.

\section{Changes in experimental design}

The underlying cause of AIS is unknown and confirmation of the diagnosis of AIS is by elimination rather than by positive means. When a patient presents with AIS, all the known causes are considered and if they are all excluded then the patient is diagnosed with AIS. An important, fundamental question in this area relates to whether or not there is a single cause for AIS development or whether there are multiple causes each with a similar outcome that 
have been included under the umbrella of AIS. The answer to this question is of paramount importance because it affects how research experiments are designed.

Patients with scoliosis have been reported and recorded since the time of Hippocrates, 2000 years ago. One might imagine his 'clinic' where there would have been patients with curved spines who would have formed a very visible group. However, identification of an underlying cause would not have been available for any of them and so they would all have been labeled simply as 'idiopathic'. As the number of patients with curved spines increased, Hippocrates would have been able to identify and isolate patients with apparently common causes and relate these causes to the curved spines. Over the years this has been shown to be true with cases of Neurofibromatosis, Friedrich's Ataxia, Poliomyelitis etc. - all such patients develop scoliosis and the underlying cause has been recognized as such. However, this culling of patients from the idiopathic group has only reduced the number of AIS patients by $20 \%$ with $80 \%$ remaining as idiopathic. It seems arrogant to suggest that there is just one, single underlying cause remaining to be found among these many patients. Perhaps it is just that the remaining causes are more difficult to identify but, nevertheless, still exist. Furthermore, scoliosis curves have tremendous variety with some being single or double, some being to the left or to the right, and some covering different levels of the vertebral column! And yet they are all included in the same category as being cases of AIS. While these differences in form may be the consequence of biomechanical differences rather than any underlying cause, they do support more the idea that there are several, if not many, separate, underlying causes of AIS yet to be discovered.

In spite of this evidence to suggest that there are many different causes of AIS, the usual experimental design found in the literature has relied on an underlying principle that there is just a single cause remaining to be found despite the facts suggesting otherwise.

A theory of scoliosis is tested by experiment. The usual experimental design consists of selecting a group of patients with AIS and a similar group of control subjects and examining the parameter in question. If there is a single cause for AIS then this experimental design might be considered satisfactory. But if there are many causes for scoliosis then this experimental design is seriously flawed because any abnormal results related to scoliosis in the experimental group of patients will be smothered by the large number of patients in whom this particular parameter is normal. For example:

- Imagine, hypothetically, that it is suspected that the underlying cause for the development of AIS is an unequal number of muscle spindles in the muscles on the two sides of the spine. This inequality has led to an unequal input of sensory information to the central nervous system which has subsequently led to a poor distribution of muscle response. This inequality has resulted in a change in force distribution and the development of a spinal curve.

- If this hypothesis were to be tested, then the logical experiment would be to collect samples of muscle tissue from the spines of patients with AIS and compare them with similar samples collected from normal subjects.

- The muscle samples would be sectioned and stained and the number of muscle spindles counted and compared between the left and right sides of the spine and between the two groups.

- If the hypothesis is correct and the imbalance in numbers of muscle spindles is the underlying mechanism responsible for all the cases of AIS, then the results table might 
look like those seen in Table 1 below. Subsequent analysis would show that there is a significant difference in the number of muscle spindles in the paraspinal muscles of patients with AIS with a significantly greater number being on the concave side. These results would suggest that there is just one underlying mechanism for the cause of AIS.

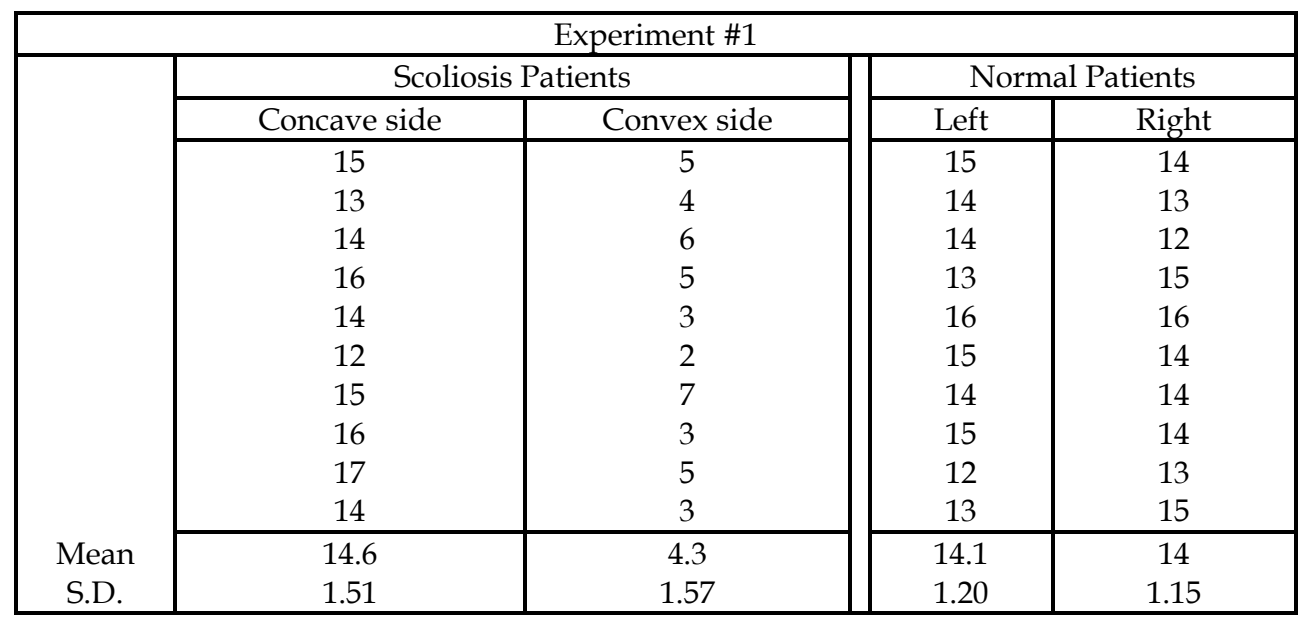

Table 1. The results from a hypothetical experiment in which the number of muscle spindles in paraspinal muscles on both sides of the vertebral column has been determined in patients with AIS and in controls. The assumption has been made that there is one underlying mechanism for the development of AIS namely, an imbalance of muscle spindle number. If such was the case, then this is the type of result that might be expected. In the scoliosis patients the number of muscle spindles on the convex side of the vertebral column is significantly less than on the concave side. In the normal patients, there is no difference in the number of muscle spindles between the two sides of the vertebral column.

- However, if there is more than one underlying cause for the development of AIS and an imbalance of muscle spindles was just one of several, then the results of a similar, but more realistic, experiment might possibly be those as shown in Table 2. Here there are two AIS patients in whom the number of muscle spindles is significantly different between the two sides of the vertebral column but their results would be masked in any statistical analysis. Such an experiment would conclude that an imbalance in the number of muscle spindles is not an underlying cause of development of AIS - which would probably be incorrect.

This experimental design is not suited for identifying single causes for the development of AIS when there are many potential causes but unfortunately many experiments relating to identification of the underlying causes of AIS have been conducted on this basis e.g. several projects looking at the levels of serum melatonin in patients with and without AIS simply compared levels in blood collected from normal subjects and from patients with AIS. While low levels of melatonin might be a cause of AIS development in some patients it probably is not the only cause. Perhaps this explains the wide variety of results to be found in the literature that are often contradictory. 


\begin{tabular}{|c|c|c|c|c|}
\hline \multirow{15}{*}{$\begin{array}{c}\text { Mean } \\
\text { S.D. }\end{array}$} & \multicolumn{2}{|c|}{ Experiment \#2 } & \multirow{2}{*}{\multicolumn{2}{|c|}{ Normal Patients }} \\
\hline & \multicolumn{2}{|c|}{ Scoliosis Patients } & & \\
\hline & Concave side & Convex side & Left & Right \\
\hline & 15 & 15 & 15 & 14 \\
\hline & 13 & 14 & 14 & 13 \\
\hline & 13 & 16 & 14 & 12 \\
\hline & 16 & $5^{*}$ & 13 & 15 \\
\hline & 14 & 13 & 16 & 16 \\
\hline & 12 & 12 & 15 & 14 \\
\hline & 15 & 17 & 14 & 14 \\
\hline & 16 & $3^{*}$ & 15 & 14 \\
\hline & 17 & 15 & 12 & 13 \\
\hline & 14 & 13 & 13 & 15 \\
\hline & 14.5 & 12.3 & 14.1 & 14 \\
\hline & 1.58 & 4.64 & 1.20 & 1.15 \\
\hline
\end{tabular}

Table 2. The results from a hypothetical experiment in which the number of muscle spindles in paraspinal muscles on both sides of the vertebral column has been determined in patients with AIS and in controls. If there is more than one underlying cause for the development of AIS then this is the type of result that might be expected. The assumption has been made that there is more than one underlying mechanism for the development of AIS e.g. muscle imbalance, ligament strength imbalance, unbalanced neural feedback, differences in growth hormone levels etc. In this scenario it is assumed that an imbalance of the number of muscle spindles between the two sides of the vertebral column is a separate cause for the development of AIS but only in some patients, not in all patients. Note that two patients reflect this imbalance $\left(^{*}\right)$. However, the analysis of the overall data would overwhelm the data from these two patients and no significant differences in average values would be found. At the end of the statistical analysis, the conclusion would be that differences in the number of muscle spindles between the concave and convex sides of the vertebral column do not contribute to the development of AIS - but this would be incorrect, at least for the two patients as shown in this hypothetical example.

Whenever an experiment is conducted as described above, the authors should be asked to explain why they selected this particular experimental design. The design is only appropriate when there is a belief that there is only one underlying cause for development of AIS and the authors should be asked to explain why they believe this is so.

While most experiments related to the aetiology of AIS are designed on the basis that there is a single underlying cause there is little reason to suspect that this is actually the case.

\section{Measurement of scoliosis curves must be made in 3-dimensions}

Traditionally, spinal curves have been measured from postero-anterior (PA) radiographs using the Cobb angle. This method has proved to be very successful especially for surgery and is useful for comparison of curve development and to present to patients to show progress. However, a radiograph is a two-dimensional (2-D) representation of a 3-D structure and all the structures in the image are compressed into a 2-D plane. As such, this is a distortion of the 3-D structure and can be easily misrepresented. Figures 3 and 4 provide a 
good example of the type of 2-D distortion that can occur when a simple, fixed, 3-D curved metal bar is rotated.

Figure 3 represents a piece of equipment in a children's playground. It consists of a single metal bar that is connected to a swivel at both the top and the bottom. The bar is curved and goes through a $360^{\circ}$ spiral along its length. The idea is for the children to turn the bar easily by simply by pulling on it and watching the spiral rotate. Apparently, this provides great entertainment. Figure 4 shows the metal bar stopped in 4 different positions (B, C, D, and E) that are each separated by $90^{\circ}$ rotation. The four images can be seen to represent the 4 most common types of curve seen in patients with AIS - single curves to the right and left (B and D) and double curves to the right and left (C and E). Therefore, from a single, curved, fixed rod different images of curved spines can be seen simply by rotation.

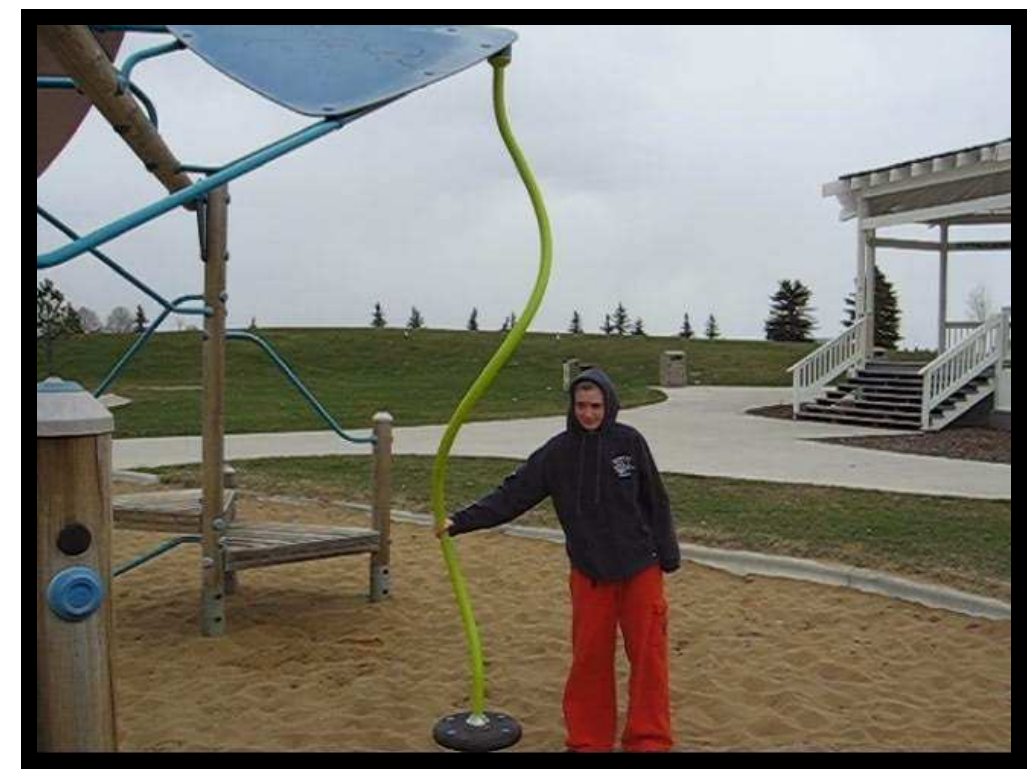

Fig. 3. A 'twirly bar' in a children's playground.

The equipment consists of a single, metal rod that curves through a complete $360^{\circ}$. It is anchored to a swivel at both the top and the bottom and can be rotated continuously simply by turning the bar.

A radiograph compresses three-dimensions into two-dimensions and the Cobb angle is consequently a two-dimensional measurement. Scoliosis curves are far from being twodimensional as they involve vertebral rotation, rotation of the ribs, and malformed vertebrae, all of which are in three dimensions. While the Cobb angle successfully provides measurement of deformity in two-dimensions, there is a great need to study curve development and improvement in three-dimensions if advances in the understanding of curve structure are to be made. If you measure in three-dimensions, you will think in twodimensions; if you measure in three-dimensions, then you will think in three-dimensions - and that is what is needed to be done. 


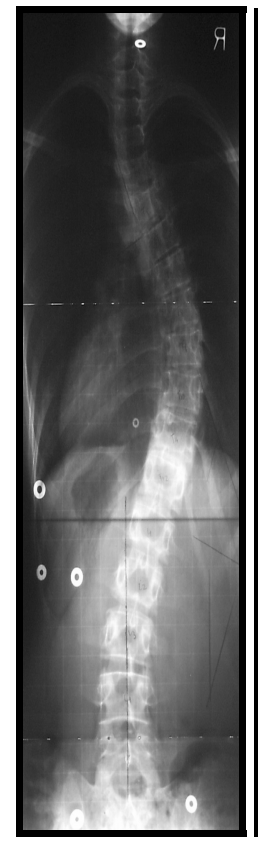

A

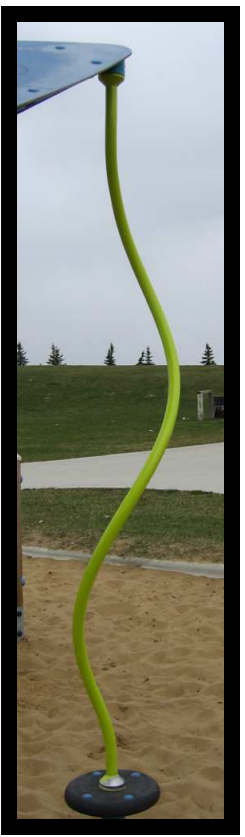

B

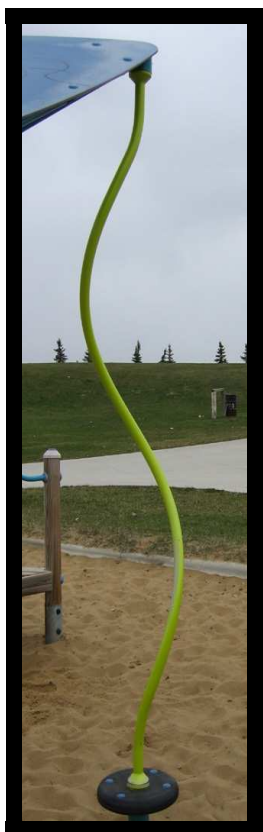

C

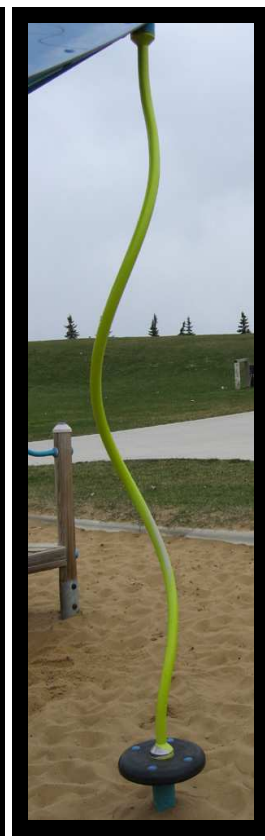

$\mathrm{D}$

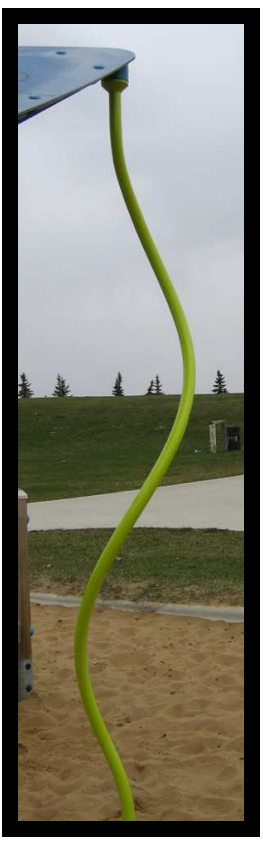

E

Fig. 4. A - a typical radiograph of a patient with AIS presented to show a resemblance to the position of the fixed, metal bar seen in B. B - the metal bar positioned to represent a single curve similar in shape to that seen in AIS with the curve being to the right. $\mathrm{C}$ - the metal bar positioned to represent a double curve in AIS with the thoracic curve being to the left. D the metal bar positioned to represent a single curve in AIS with the curve being to the left. E - the metal bar positioned to represent a double curve in AIS with the thoracic curve being to the right. Overall, the four major curves commonly seen in AIS can be represented by the same fixed curve viewed in different positions. This sequence highlights the importance of rotation being a major factor in the analysis and evaluation of AIS from 2-D radiographs.

The measurement of a spinal curve in 2-D from a PA radiograph is easy to make and easy to understand, especially for the patients and their relatives: 'Six months ago your curve was $30^{\circ}$ and now it is $20^{\circ}$. The treatment is effective and your curve is being reduced.' There are considerable errors associated with taking radiographs of curved spines and measuring the Cobb angle but these are well-recognised and much effort has been made to overcome them especially those associated with consistent positioning and observer errors. However, as Figures 3 and 4 have shown, a simple rotation of the spine can produce a completely different image. This change in image can easily be mistaken for a change in the structure of the spinal curve (e.g. a decrease/increase in curvature) where none has actually occurred. A spinal curve is a 3-D structure and that fact cannot be ignored. No amount of 2-D measuring can overcome this basic issue.

A 3-D curve is a much more complicated structure to measure than a 2-D curve and the problem now arises as to how to keep such measurements simple for communication 
purposes. A 3-D curve is perhaps best represented by an equation which can be calculated quite easily using a 3-D coordinate system. A simple example might be:

$$
A=3 x y^{3} z^{2}+x^{3} y z^{3}+x^{2} y^{2} z
$$

While this might depict accurately the shape of the curve in its present form, it is much more difficult to understand than a simple number of degrees (e.g. $30^{\circ}$ ) as is presented with the 2$\mathrm{D}$ measurement. It is also difficult to understand how a change in such an equation can be made to be meaningful especially to the patients and to their relatives who simply want to know whether the curve has got better or worse. For example, analysis of the same spinal curve on a subsequent visit might reveal an equation such as:

$$
A=4 y^{2} z^{4}+10 x^{2} y z^{3}+3 x^{2} y^{2}
$$

and it is difficult to provide a method whereby the subtle changes between the two equations could be expressed clearly to the patient!

Methods for producing 3-D images of scoliosis spines are becoming more readily available. Methods for describing these curves in 3-D should receive high priority because of their usefulness in providing a more accurate description of the spinal curve and any changes that might be involved. While the 2-D measurement provided by the Cobb angle has been useful in the past and remains useful to the clinician, if significant advances are to be made in scoliosis research, there has to be a shift in attitude and 3-D measurement should become routine and used as the gold standard.

\section{The promise of genetic studies}

The rapid development of genome-sequencing techniques in recent years has reduced the time to sequence the genome of a patient to a matter of just a few days. For the past few years, utilizing this ability has led to much research into many diseases being focused on selecting patients with a common problem and analyzing the genetic make-up of such patients to identify problem genes. In some diseases this has been very successful, especially where a single problem gene has been able to be identified. Such techniques have been applied to patients with AIS but, unfortunately, the picture that has emerged does not seem to be a simple one. Genetic research into AIS has shown many genes possibly to be involved and the picture is most certainly complex even though diagnostic tests are currently being developed (Akoume et al, 2010; Leboeuf et al., 2009; Ogilvie, 2010; Weinstein et al, 2008). This complexity might well reflect the complex nature of AIS (as described above) and the mixed nature of patients with possibly very different underlying causes. Alternatively, it might well describe the multifactorial nature of AIS with many factors being involved even in just a single case. Whatever the outcome, the results from the genetic studies of AIS seem to have the greatest potential for providing a solution and much better understanding of the problem. In addition, the results from such studies would provide much needed guidance to many other areas of research.

One aspect of the complexity of AIS has always been the question of whether all cases have a similar underlying cause (as discussed earlier) or whether the patients represent similar manifestations of a variety of different causes. The lack of resolution of this problem has had repercussions for AIS research. For example, in the past a group of patients with AIS 
selected for research might have included both males and females, patients with single or double curves, patients with curves to the left or to the right, curves that span just a few vertebrae, curves that span many vertebrae, curves of just a few degrees, much more severe curves, curves that have been present for a long time, curves that have been present for a short time etc. The only common feature in selection of such a group is that they all fall under the umbrella of AIS with all its apparent diversity. Such a selection process leaves much to be desired and it is not difficult to recognize the inherent problems within such a group especially if there are several different underlying causes which are different for each case and even more so if there are multiple factors contained within those different underlying causes. The selection of such groups is not ideal but is often made by necessity with a lack of numbers of patients having to be overcome to make statistical analysis worthwhile.

With genetic research, the opportunity to overcome such difficulties has been presented because of the intricacy of the work. To overcome or simplify the issue of there being many different causes of AIS and consequently reduce the number of potential genes involved, many genetic studies are now looking at Familial Idiopathic Scoliosis (FIS) where only patients from the same family are being investigated. In theory, this should narrow the scope of investigation to a single (albeit multifactorial) underlying cause but even here, from the results so far, there are still many genes that appear to contribute to the abnormal development. However, at least it is a step in the right direction and should be encouraged.

The concept of the underlying cause of AIS being multifactorial is also important to consider in this context, even within the family environment. Interestingly, research has shown that if a patient has AIS there is a $\sim 10 \%$ probability that a sibling will also develop AIS. This probability is too high for this second development of AIS to be random and so AIS is referred to as being familial i.e. it can be found in several people within the same family.

In the case of identical (monozygotic - identical DNA) twins, it has been shown that there is only a $\sim 70 \%$ probability of both twins developing scoliosis. This is important because it highlights the impact of factors other than genetic make-up in developing scoliosis and has spawned the concept of epigenetics where factors other than genetics are considered to be major contributing factors (Grivas et al., 2002a, 2002b, 2006, 2007, 2008a, 2008b, 2009, 2001). Factors that might be considered must certainly include environmental conditions and their influence on spinal curve development and might present alternative treatment strategies (Hermus et al., 2007). This probability of $\sim 70 \%$ also shows the limitations of genetic screening being successful in this search because individuals with identical DNA can have diverse degrees of scoliosis, sometimes ranging from severe in one twin to none in the other. This is a dilemma that needs to be resolved and would be an important key in the solution to the problem.

Most interesting though is the finding that for non-identical twins (dizygotic - with nonidentical DNA that merely has sibling similarity) there is a $\sim 35 \%$ probability that they will both develop scoliosis. Non-identical twins are nothing more than siblings who have shared a common uterus at the same time. This would suggest that the probability of both twins getting scoliosis should be $10 \%$ and not the $\sim 35 \%$ as is found. It remains to be seen what complexities exist in relation to the uterine environment that might create this increased 
probability. Again, this is a dilemma of genetic studies that remains to be resolved and one that requires focus if greater progress is to be made

\section{Development of computer-assisted methods of research}

The development of scoliosis is a slow process and takes several years in humans. There are very few reliable and successful animal models that approach being able to replicate the development of AIS in humans and even these take several weeks if not months for any curve to become manifest. The use of computer-assisted methods and models to mimic the curve development are essential research tools to be developed because their application would have many advantages:

- The time for development of the scoliosis curves would be greatly reduced and results could be seen in hours rather than weeks and months.

- The development of such methods would eliminate/reduce the need for animal research

- Such methods would allow for many different methods and ideas to be tested quickly and simply. The quick results could also enable alternative ideas and thoughts to be tested immediately.

- The results from such methods would allow for a better understanding of the mechanics and underlying mechanisms for the development of spinal curves

- The methods would allow for the development of better treatment strategies which could be applied before actual treatment to predict results.

However, currently the time required for the development of computer-assisted modeling is long and very time-consuming. Furthermore, many practical results relating to tissue parameters are still required to provide adequate and accurate input data and collection of these data all requires time. However, the benefits of being able to use computer assisted modeling to view curve development quickly are well worthwhile. Moreover, the extended application of developing treatment strategies and viewing their predictive value prior to their actual application would be immense (Driscoll et al., 2010, 2011a, 2011b).

\section{Is anybody looking for a cure?}

In his recent book (Where are all the leaders?), Lee Iacocca, the former, very successful CEO of Chrysler reported on his retirement activities. Having tried all the usual activities such as playing golf he realized he was bored and so focused on raising funds for research for a health-related charity. Again, he was very successful but after a while he realized something that he thought influenced the research world. He came to the conclusion that research is never-ending and has become an industry all on its own. In effect: 'Researchers get grants to do research so that they can write papers to get more grants to do more research so that they can write more papers to get more grants to do more research ....... and on it goes.' In the book he asks quite simply: 'Is nobody looking for a cure?' How many people are looking for a cure for AIS?

It would be a great relief if a cure for AIS could be announced tomorrow. If a means by which a spinal curve could be prevented from developing as well as a cure for all those patients who already have a curve were to be announced it would be incredible. If that happened then people involved with research into the aetiology of AIS would be very excited and all the effort that is being put into research of AIS could be channeled elsewhere. 


\section{Conclusion}

An understanding of scoliosis involves an enormous amount of interest and covers many areas of academia. The ideas and thoughts related to AIS in particular are scattered in many different directions. This chapter has attempted to identify those areas of research where most progress towards a better understanding of scoliosis might be made. This is not to say that other areas of research in scoliosis have less value but the chapter has tried to identify those areas where most progress can potentially be made and where focus should be directed. Whether this can be achieved as such remains to be seen.

\section{References}

Akoume, M, Azeddine, B, Turgeon, I, Franco, A, Labelle, H, Poitras, B, Rivard, C., Grimard, G, Ouellet, J, Parent, S \& Moreau, A. (2010) Spine. Cell-based screening test for idiopathic scoliosis using cellular dielectric spectroscopy. 35, pp. E601-608.

Driscoll, M, Aubin, C, Moreau, A, \& Parent, S. (2011a) Biomechanical comparison of fusionless growth modulation corrective techniques in pediatric scoliosis. Med Biol Eng Comput (Epub ahead of print).

Driscoll, M, Aubin, C, Moreau, A, \& Parent, S. (2011b) Spinal growth modulation using a novel intravertebral epiphyseal device in an immature porcine model. Eur Spine J (Epub ahead of print).

Driscoll, M, Aubin, C, Moreau, A, \& Parent, S. (2010) Finite element comparison of different growth sparring instrumentation systems for the early treatment of idiopathic scoliosis. Stud Health Technol Inform 158, pp 89-94.

Grivas, TB, Dangas, S, Polyzois, BD, \& Samelis, P. (2002a). The Double Rib Contour Sign (DRCS) in lateral spinal radiographs: aetiologic implications for scoliosis. Stud Health Technol Inform. 88, pp.38-43.

Grivas TB, Dangas S, Samelis P, Maziotou C, \& Kandris K. (2002b) Lateral spinal profile in school-screening referrals with and without late onset idiopathic scoliosis 10 degrees-20 degrees. Stud Health Technol Inform. 91, pp.25-31.

Grivas, TB, Vasiliadis, E, Malakasis, M, Mouzakis, V., \& Segos, D. (2006) Intervertebral disc biomechanics in the pathogenesis of idiopathic scoliosis. Stud Health Technol Inform. 123, pp.80-3.

Grivas TB, Vasiliadis ES, Mihas C, \& Savvidou O. (2007) The effect of growth on the correlation between the spinal and rib cage deformity: implications onidiopathic scoliosis pathogenesis. Scoliosis. Sep14, pp.2-11.

Grivas, TB, Vasiliadis, ES, Mihas, C, Triantafyllopoulos, G \& Kaspiris, A. (2008) Trunk asymmetry in juveniles. Scoliosis. Sep 23, pp.3-13.

Grivas TB, Vasiliadis ES, Rodopoulos G, \& Bardakos N. (2008a) The role of the intervertebral disc in correction of scoliotic curves. A theoretical model of idiopathic scoliosis pathogenesis. Stud Health Technol Inform. 140, pp.33-6.

Grivas TB, Vasiliadis E, Savvidou OD, \& Triantafyllopoulos G. (2008b) What a school screening program could contribute in clinical research of idiopathic scoliosis aetiology. Disabil Rehabil.;30(10):752-62. Review.

Grivas TB, Burwell RG, Mihas C, Vasiliadis ES, Triantafyllopoulos G, \& Kaspiris A. (2009) Relatively lower body mass index is associated with an excess of severe truncal asymmetry in healthy adolescents: Do white adipose tissue, leptin, hypothalamus 
and sympathetic nervous system influence truncal growth asymmetry? Scoliosis. Jun 30, pp.4:13.

Grivas TB, Vasiliadis ES, Kaspiris A, Khaldi L, \& Kletsas D. (2011) Expression of matrix metalloproteinase-1 (MMP-1) in Wistar rat's intervertebral disc after experimentally induced scoliotic deformity. Scoliosis. May 9, pp.6(1):9.

Hermus, J, Rhijn, L, Ooij, A. (2007). Non-genetic expression of adolescent idiopathic scoliosis: a case report and review of the literature. Eur Spine J. 16 (suppl 3) 338341.

Keim, H. (1979) Scoliosis. CIBA Clinical Symposia. Vol 31, number 2.

Leboeuf, D, Letellier, K., Alos, N, Edery, P, \& Moldovan, F. (2009) Do estrogens impact adolescent idiopathic scoliosis? Cell. 20, 4, pp147-152.

Ogilvie, J. (2010). Adolescent idiopathic scoliosis and genetic testing. Curr Opin Pediatr. 22 (1), 67-70.

Weinstein, S, Dolan, L, Cheng, J, Danielsson, A \& Morcuende, J. Adolescent idiopathic scoliosis. (2008) The Lancet. 371, pp. 1527-1537. 


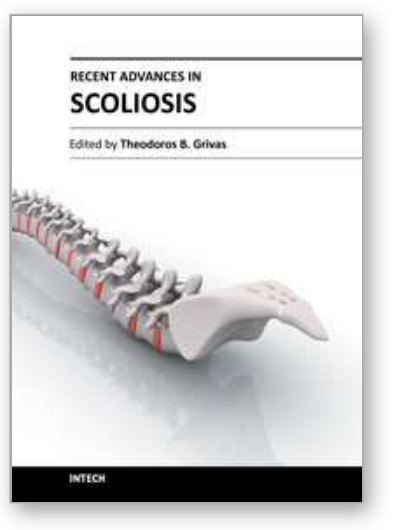

\author{
Recent Advances in Scoliosis \\ Edited by Dr Theodoros Grivas
}

ISBN 978-953-51-0595-4

Hard cover, 344 pages

Publisher InTech

Published online 09, May, 2012

Published in print edition May, 2012

This book contains information on recent advances in aetiology and pathogenesis of idiopathic scoliosis, for the assessment of this condition before treatment and during the follow-up, making a note of emerging technology and analytical techniques like virtual anatomy by 3-D MRI/CT, quantitative MRI and Moire Topography. Some new trends in conservative treatment and the long term outcome and complications of surgical treatment are described. Issues like health related quality of life, psychological aspects of scoliosis treatment and the very important "patient's perspective" are also discussed. Finally two chapters tapping the untreated early onset scoliosis and the congenital kyphoscoliosis due to hemivertebra are included. It must be emphasized that knowledgeable authors with their contributions share their experience and enthusiasm with peers interested in scoliosis.

\title{
How to reference
}

In order to correctly reference this scholarly work, feel free to copy and paste the following:

Keith M. Bagnall (2012). How to Improve Progress in Scoliosis Research, Recent Advances in Scoliosis, Dr Theodoros Grivas (Ed.), ISBN: 978-953-51-0595-4, InTech, Available from:

http://www.intechopen.com/books/recent-advances-in-scoliosis/where-to-focus-to-improve-scoliosis-research

\section{INTECH}

open science | open minds

\section{InTech Europe}

University Campus STeP Ri

Slavka Krautzeka 83/A

51000 Rijeka, Croatia

Phone: +385 (51) 770447

Fax: +385 (51) 686166

www.intechopen.com

\section{InTech China}

Unit 405, Office Block, Hotel Equatorial Shanghai

No.65, Yan An Road (West), Shanghai, 200040, China

中国上海市延安西路65号上海国际贵都大饭店办公楼 405 单元

Phone: +86-21-62489820

Fax: $+86-21-62489821$ 
(C) 2012 The Author(s). Licensee IntechOpen. This is an open access article distributed under the terms of the Creative Commons Attribution 3.0 License, which permits unrestricted use, distribution, and reproduction in any medium, provided the original work is properly cited. 